Revista Meridiano. No. 32. CEPUN, Universidad de Nariño. (ISSN: 0121-0038). (pp. 250-258). 1996.

APA citation style: Benavides B., Jorge E. (1996). Hacia una Política para el Uso del Computador en Educación. Revista Meridiano, Universidad de Nariño (32), 250-258.

\title{
Hacia una Política para el Uso del Computador en Educación
}

\author{
Jorge E. Benavides B. (joelbebu@gmail.com) \\ Departamento de Lingüística e Idiomas \\ Universidad de Nariño, San Juan de Pasto, Colombia
}

\section{Introducción}

Para utilizar la tecnología de la información (informática aplicada o informática educativa) a través de un determinado currículo, se necesita una idea clara de lo que se puede lograr, es decir lo que es y no es realizable. Además, se necesita comprender para qué son buenos los computadores, sus capacidades y limitaciones en educación. Esto solo se puede conseguir cuando los profesores se concienticen de las áreas de uso más importantes y del software (programas de computador) disponible. Sin embargo, no sería real, ni siquiera deseable para el desarrollo de una política de uso de la tecnología educativa, el determinar objetivos precisos en todas las materias y áreas del conocimiento en este momento, ya que esto solo se lograría con la decisión del profesor, personal administrativo y estudiantes en el tiempo.

Esta política debería solo sugerir o delinear los objetivos generales, las razones para la inclusión de la tecnología educativa dentro del currículo, las estrategias de tipo general y las habilidades y destrezas específicas a desarrollarse. También deberían delinear las formas en las que la tecnología de la información puede utilizarse.

\section{Contextualización}

Es importante destacar que no muy pocas instituciones de carácter oficial y privado de nivel pre-escolar, primario y de bachillerato han hecho adquisición de computadores para utilizarse como medio de instrucción. La indicación es que estas y otras instituciones continuaran invirtiendo en forma importante en la nueva tecnología. Esta inversión incluye solamente la compra de equipos y programas (hardware y software) sin embargo se está olvidando una parte crucialmente importante para el uso efectivo de esta tecnología educativa: el entrenamiento de profesores en este campo.

La disponibilidad de estos equipos de ninguna manera debe constituirse en garantía de que los estudiantes harán el mejor uso o que se mejoraría de alguna manera la calidad de la educación. La clave de que esta introducción funcione está en que se tenga (acuerde) 
Revista Meridiano. No. 32. CEPUN, Universidad de Nariño. (ISSN: 0121-0038). (pp. 250-258). 1996.

una política pedagógica sobre el uso del computador entre las instituciones, profesores, padres de familia y estudiantes de una sociedad.

\section{Cómo identificar el buen uso del computador}

En educación, los computadores pueden utilizarse en una cantidad variada de formas y para diferentes propósitos (Benavides, 1989, 1993), y las primera tarea que tienen los profesores es la de identificar las aplicaciones que los estudiantes harían y de las cuales se beneficiarían (es indudable que en el momento los computadores pueden estar siendo utilizados para tareas no muy practicas con los estudiantes). Por ejemplo, algunos de los primeros programas en el mercado se basan en la idea de que los computadores estaban diseñados para ayudar al estudiante a aprender conceptos y hechos específicos. De esta manera se tiene el concepto de que el programa significaba solo una especie de hoja electrónica, permitiendo la práctica de destrezas básicas o dando lugar a actividades de refuerzo. Sin embrago, esta clase de programas utilizaba muy poco del poder (velocidad, memoria, etc.) del computador y de esta manera las actividades no estaban de acuerdo a las necesidades de los estudiantes. Aspectos como lo atractivo de los gráficos y la animación podrían cautivas la atención por un poco tiempo pero no constituyen en si el propósito de la actividad o de la aplicación en situaciones reales y con resultados significativos. Afortunadamente el software educativo está progresando y mejorándose cada vez más y una gran cantidad está disponible en nuestros días, sobre todo por la cercanía relativa con países productores de estos programas (México, Estados Unidos, Chile, etc. y, desde luego, la incipiente producción de buen software educativo colombiano)

Con el anterior orden de ideas, es importante mencionar que una filosofía ya sea intra o interinstitucional debe prevalecer sobre la relativa atracción de una determinada pieza de software. Por otro lado, una de las propiedades de esta filosofía debería impulsar la 
Revista Meridiano. No. 32. CEPUN, Universidad de Nariño. (ISSN: 0121-0038). (pp. 250-258). 1996.

hipótesis de que se utilice el computador cuando su velocidad, potencia, calidad de gráficos, interactividad, etc., mejoren la calidad del trabajo que se realice.

Por supuesto que la anterior hipótesis es solo una parte de la filosofía en general, la cual necesitaría más dedicación y clarificación. Se necesitaría pues vislumbrar algunos de los objetivos generales y específicos.

\section{Objetivos generales y ventajas de una política de uso del computador}

1. Una política del uso del computador en educación debe encaminarse e introducir a los estudiantes (niños, pre-adolescentes, adultos) en la forma cómo se utilizan los computadores en la sociedad. Indudablemente que los computadores son una parte de la actividad diaria de la vida moderna, y, como tal, los niños, por ejemplo, deberían tener confianza al utilizarlos y entender las implicaciones de su uso.

2. Hay un gran número de destrezas y habilidades que los niños necesitan desarrollar si desean hacer uso efectivo del computador. Estas no deben verse en aislamiento sino dentro del mismo currículo. Uno de los más importantes se relacionan con el procesamiento de texto.

El estudiante que es capaz de utilizar un procesador de texto será capaz de aplicar ese uso no solamente para escribir cuentos, historias o poemas, sino también para preparar reportes de las demás materias, como en ciencias, u organizar oraciones (ideas) preparando una exposición o charla a otros estudiantes.

El resultado de la revisión del trabajo de un estudiante podría dar una buena pieza de texto escrito, el cual no solamente refleja la habilidad en sí misma sino su presentación en forma apropiada. 
Revista Meridiano. No. 32. CEPUN, Universidad de Nariño. (ISSN: 0121-0038). (pp. 250-258). 1996.

3. Esta política de uso de los computadores en educación debería permitir que los estudiantes desarrollen un pensamiento lógico, una mentalidad orientada al desarrollo de problemas, y técnicas de control que les permitan responsabilizarse de su propio aprendizaje con eventual desarrollo de su autonomía (aprendizaje autónomo). La naturaleza interactiva de ciertos programas, como LOGO, con los niños significa que estos pueden progresar en cuanto a procesos de aprendizaje a su propio ritmo, reduciendo problemas grandes a procesos más manejables (Papert, 1980) y construyendo un mecanismo más viable hacia las soluciones de problemas.

4. El papel y poder modelador del computador debería utilizarse para estimular los ambientes más allá del alcance normal del salón de clases. Existen paquetes (programas o software) excelentes de simulación y aventuras que permiten crear ambientes seguros donde los niños pueden explorar problemas (aprendizaje exploratorio) dentro de un grupo o trabajando con este para encontrar una solución compartida (aprendizaje colaborativo) de forma que no sería posible en un contexto normal de desarrollo de clase (aprendizaje tradicional).

5. El uso de hardware y software específico y relevante, deberían ir de la mano con las necesidades de un individuo o grupo de individuos. Todavía existe un lugar importante para software de propósito específico que utiliza la naturaleza interactiva del computador para asistir al niño o joven o grupos de estos con un aspecto particular de su trabajo. Esta clase de software podría, por ejemplo, utilizarse para reforzar la habilidad de interpretar un gráfico, o explorar la simetría de un objeto.

Podría habilitar a los estudiantes con problemas específicos de aprendizaje para manejar una habilidad particular de una manera motivante y sin sentirse amenazados o intimidados. Este tipo de software requerirá a menudo de otros 
Revista Meridiano. No. 32. CEPUN, Universidad de Nariño. (ISSN: 0121-0038). (pp. 250-258). 1996.

materiales de apoyo y necesidades para ultimarse con cuidado, sobre todo si se desea que los estudiantes se beneficien al máximo.

6. Esta política debería desarrollar un uso amplio de los computadores a través del currículo y mejorar el trabajo ya alcanzado hasta el momento sin su uso. En este orden de ideas, los profesores podrían argumentar que no disponen de tiempo dentro del currículo para añadir una materia más. Sin embargo, el computador no debe mirarse como un nuevo tópico o materia sino como otra posibilidad de trabajo dentro o fuera del aula de clases.

El procesamiento de texto no debe mirarse como un dominio de la lengua o el trabajo con base de datos como un trabajo solo de las matemáticas. Solamente cuando el computador sea visto como una herramienta o instrumento utilizado dentro del currículo, su integración dejará de ser fragmentaria y los estudiantes se beneficiarían de este uso.

\section{Ventajas del uso del computador}

- Algunos de los beneficios del uso del computador ya se han mencionado, pero una visión más clara podría aportar más criterios para que los profesores evalúen y decidan si están o no haciendo el mejor uso de él. Los computadores aportan motivación (altas validez física). Cualquier persona que haya utilizado computadores con niños sabrá qué difícil es separarlos del teclado ya sea que estén trabajando en una actividad de grupo, escribiendo una historia o practicando una habilidad en particular.

- El hecho de utilizar computadores les da a los estudiantes un buen sentido de logro, especialmente en el área del procesamiento de texto donde el resultado final puede ser una pieza perfecta. La retroalimentación también puede ser inmediata ya que, en el caso del computador, con el estudiante no se necesita tanto esperar a que el profesor acuda en su ayuda. 
Revista Meridiano. No. 32. CEPUN, Universidad de Nariño. (ISSN: 0121-0038). (pp. 250-258). 1996.

- En el caso de los niños, por ejemplo, estos pueden evaluar y corregir su propio trabajo en áreas diferentes a las propias del procesamiento de texto. Cualquier buen software que coloca al niño en control de su propio aprendizaje debe ser capaz de hacer esto. Dibujar una silueta, utilizando gráficas, sería un proceso constante de intentar algo y luego mejorarlo. Esto no es siempre fácil de lograr en otras situaciones de clase.

- Utilizar un computador permite obtener resultados que se pueden presentar en una gran cantidad o variedad de formas. Cuando se utiliza un procesador de texto, es sencillo alterar la conformación de una página o seleccionar el estilo de impresión más apropiado. En forma similar, cuando se utiliza software de base de datos, las gráficas pueden dibujarse y volverse a dibujar, alterarse las escalas y cambiarse los grupos de frecuencias para obtener la conformación o despliegue de los datos más apropiados.

- La velocidad del computador ayudará a los estudiantes a llegar a ser más eficientes para manejar y recuperar los datos. Tareas que habrían tomado mucho tiempo al hacerlas a papel y lápiz, se logran fácilmente permitiendo que los estudiantes mantengan el interés en la interpretación de los resultados más que en la mecánica de despliegue de la información.

- Los computadores permiten lograr simulaciones de experiencias, las cuales serían imposibles de obtener de otra manera. Buscar un barco hundido o realizar una exploración arqueológica, una caída al resbalar, no son eventos de todos los días, pero el computador puede proporcionar una experiencia segura al estudiante sobre los problemas y decisiones que se contemplan.

- El uso del computador impulsa el desarrollo de habilidades y estrategias de grupos, que son rara vez utilizadas por el estudiante solo. Esto motiva a los estudiantes a discutir su trabajo y llegar a decisiones de grupo acerca del mejor o más apropiado curso de acción, o sobre las preguntas más apropiadas para obtener la información 
Revista Meridiano. No. 32. CEPUN, Universidad de Nariño. (ISSN: 0121-0038). (pp. 250-258). 1996.

que se necesita de una base de datos; en pocas palabras, se contempla la ocurrencia del aprendizaje colaborativo.

- Estas ventajas son importantes, sin embargo deben tenerse en cuenta en relación con el desarrollo general o integral del estudiante. En el caso del software de simulación, por ejemplo, éste nunca debe reemplazar la experiencia de primera mano cuando ésta disponible.

\section{Objetivos específicos}

Una vez identificados los objetivos generales y las ventajas del uso del computador, es posible considerar de cerca áreas más específicas posiblemente con la intención de identificar habilidades y destrezas particulares o conceptos que los estudiantes deberían adquirir.

Una lista de objetivos podría ser útil para que los profesores tengan una idea clara de los objetivos de los establecimientos educativos y también como medio para asegurar un desarrollo estructurado de las habilidades de los estudiantes durante sus años estudio, ya sea a nivel pre-escolar, básica primaria o básica secundaria.

Una de las áreas en las que podemos identificar una serie de objetivos se requiere al desarrollo de habilidades de procesamiento de texto (John \& Forescue, 1987).

1. Habilitar a los estudiantes y profesores a iniciar y editar textos en forma más fácil.

2. Asistir en el desarrollo de habilidades secuenciales.

3. Motivar a los estudiantes a escribir para una audiencia más amplia.

4. Permitir a los estudiantes que produzcan trabajos de presentación consistente.

5. Habilitar a los estudiantes a producir una copia perfecta de su propio diseño. 
Revista Meridiano. No. 32. CEPUN, Universidad de Nariño. (ISSN: 0121-0038). (pp. 250-258). 1996.

En el caso de los niños de primaria, al terminar éstos su proceso de estudios, la mayoría de estudiantes deberían estar en capacidad de utilizar un procesador de texto para escribir e imprimir su propio trabajo. Esto integraría las siguientes habilidades.

1. Preparación: alistar el computador para realizar procesamientos de texto.

2. Introducción de texto: control del cursor, familiaridad con el teclado y corrección de caracteres.

3. Edición: movimiento y manipulación de texto además de búsqueda sencilla de palabras.

4. Formato: márgenes y longitud de página, justificación y centrado.

5. Impresión: uso de la posición correcta del papel, calidad y estilo de impresión.

Estos objetivos pueden, a su vez, especificarse más aún para incluir etapas particulares dentro de destrezas y habilidades. De esta manera se pueden identificar ciertas habilidades de teclado.

1. Selección de opciones de escritura desde el software

2. Habilidades de teclado básicas, es decir, mayúsculas, minúsculas, símbolos barra espaciadora y las teclas de retroceso (borrado) y de entrada (introducción) (BACKSPACE y ENTER).

3. Movimiento de las teclas del cursor dentro de la pantalla letra por letra (o carácter por carácter), palabra por palabra, línea por línea, párrafo por párrafo, página por página, al comienzo y al final del documento.

Esta clase de objetivos puede ayudar a identificar programas de computador específicos apropiados para diferentes tipos de edades y actividades. Por ejemplo, programas como LOGO son ideales para niños, pero no son apropiados para diseñar tablas de resultados en ciencias o para escribir una carta formal. 
Revista Meridiano. No. 32. CEPUN, Universidad de Nariño. (ISSN: 0121-0038). (pp. 250-258). 1996.

\section{Bibliografía}

Benavides B., Jorge E. (1989). Iniciación a la Instrucción Complementaria del inglés mediante Computadores. Revista de Investigaciones. Universidad de Nariño. No. 5, vol. III. 179-193.

Benavides B., Jorge E. (1993). Informática Educativa para la Enseñanza de los Idiomas. Revista de investigaciones. Universidad de Nariño. Año 7, No. 1, vol. VI. 196-206.

Benavides B., Jorge E. (1993). Desarrollo de la velocidad de la lectura y vocabulario mediante el uso de materiales informáticos. Revista de Investigaciones "Criterios" Universidad Mariana. 1(2), 38-58.

Papert, S. (1980). Mindstorms: Children, Computers, and Powerful Ideas. New York: Basic Books.

Jones, A. \& S. Fortscue (1987). Using computers in the language classroom. London: Longman. 